\title{
Analysis of admissions to the pediatric emergency ward of Tikur Anbessa Hospital in Addis Ababa, Ethiopia
}

\author{
Dagnew Muluneh ${ }^{1}$, Damte Shimelis ${ }^{2}$, Daniel Benti ${ }^{3}$
}

\begin{abstract}
Background: The trend of diseases varies across time depending on the change in human health behavior and life style, environmental factors and epidemiology of diseases. Health facility-based studies can give us proxy information on the burden of diseases in the community. Knowing the important causes of childhood morbidity and mortality enables planners to design proper priority setting and intervention planning.

Objective: To analyze the characteristics of patients admitted under pediatric emergency ward of Tikur Anbessa Hospital and identify important causes of admissions and deaths.

Methodology: A cross-sectional retrospective analysis of admissions to the pediatric emergency ward of Tikur Anbessa hospital was made for a period of one year from September 2002 to August 2003.

Result: A total of 2,522 patients were admitted in one year, among whom 1,459 (57.9\%) were males and 1,063 (42.1\%) were females with a male to female ratio of 1.4. The mean age of the study subjects was 2.8 years for males and 2.9 years for females. For both sexes the maximum age was 13 years and $76.5 \%$ of patients were 5 years of age or less. Out of 2,522 admissions, 360 (14.3\%) died in the emergency ward. Severe pneumonia accounted for $38.3 \%$ of the total admissions and $41.9 \%$ of the deaths.

Conclusion: Interventions targeting prevention of pneumonia, sepsis and meningitis for children will have paramount importance in reducing childhood illnesses and deaths in the country. Since most of the causes of death in the ward are treatable, there is a room to reduce mortality by improving early case detection and management. [Ethiop.J.Health Dev. 2007;21(1):48-52]
\end{abstract}

\section{Introduction}

Disease distribution is a function of several factors that influence its occurrence. (1) Most important elements which are necessary for occurrence of disease transmission are existence of susceptible population, disease causing organism and means of transmission. Any change in the magnitude of these agents affects the prevalence or incidence of diseases in the community. The emergence of new pathogens and implementation of health promoting intervention programs that influence human behavior are also important factors that affect the transmission of disease.

Most of the routine disease epidemiology relies on reports of notifiable diseases. Using such figures cases may be compared over time or between regions and countries (1). Childhood deaths in the last century reportedly decreased because of medical intervention. However, Ethiopia is still one of the six countries in the world where half of childhood deaths occur (2). In the Butajira Project, a field study conducted in 1987 in southern Ethiopia, the estimated under-five mortality and infant mortality rates were 293/1000 and 136/1000, respectively (3). In most developing countries notifiable diseases are under-reported and the data are usually based on case reports. Also, the reporting system in Ethiopia is not satisfactory enough to produce good quality information. $(4,5)$ Analysis of hospital admissions will be helpful to fill the information gaps on disease epidemiology that exist in the routine disease reporting system in developing countries(6).
The Pediatric Department of Tikur Anbessa Hospital (TAH) in Addis Ababa has ten specialty clinics with weekly visits of each and an outpatient department (OPD). It has also diarrhea treatment and expanded program on immunization (EPI) units.

All acutely ill children who need hospitalization, excluding neonates $<7$ days of age and surgical emergencies that need urgent intervention, are first admitted to the Emergency Ward. This analysis is confined to the casualty ward and did not include patients admitted to other wards or treated in the OPD.

In TAH the physician consultation and treatment cost is affordable to most people in the city as compared to private clinics. In addition, the pediatric department of the Hospital serves all patients from all corners of the city with out requesting any referral paper and hence the data in the emergency unit can give us good estimate on the important causes of emergency childhood problems in the pediatric age group of the population in the area under consideration.

The objective of this study is to analyze the characteristics of patients admitted under the emergency unit of TAH and identify important causes of admission and deaths.

The study findings can also be compared with those of previous studies and can give sound information if there is any change in disease distribution in the past years. The findings can also be used to direct important areas of

${ }^{1}$ Bahirdar Hospital; ${ }^{2}$ Department of Pediatrics, Faculty of Medicine, Addis Ababa University, Addis Ababa, Ethiopia, E-mail: damte_shimelis@yahoo.com, ${ }^{3}$ WHO Ethiopia, E-mail: dbenti2003@yahoo.com, Tel. 0911406593 
further investigation to know causes of childhood diseases.

\section{Methods}

Tikur Anbessa Hospital is a University referral hospital located at the center of Addis Ababa. All other departments except pediatric department serve mainly referred patients. The pediatric department serves for any under fifteen children who seek treatment in the Hospital. In this study, children who came seeking for medical treatment and admitted in the emergency ward of pediatric department from September 2002 to August 2003 are the study subjects. Furthermore, all patients who came to the Pediatric Emergency ward and registered with any diagnosis during admission or discharge in the study period were included. The Pediatric Emergency ward of TAH is located on the first floor of the hospital premises and is usually crowded with admitted patients and emergency treatment. Most of the time it is very common to find the corridor full of admitted patients and other pediatric patients with emergency problem waiting for their turn.

A cross-sectional retrospective study was conducted at the Emergency Ward of the Pediatrics Department of Tikur Anbessa Hospital. Data were collected by medical students who were trained for five hours. A pre-tested questionnaire was used to collect data from the ward registers for all children admitted to the emergency ward from Sep. 2002 to Aug. 2003. The Principal Investigator was continuously supervising the data collectors and $10 \%$ of the records were cross checked against hospital medical records. The questionnaire contains items on demographic data (age, sex, and address), admission date, discharge date, admission diagnosis and diagnosis at discharge or death. If address was not properly recorded on the patient registry that case was excluded from the analysis. Date of admission was not recorded for those patients who died in the ward and therefore it was not possible to know how long they stayed in the ward before they died. In cases where more than one diagnoses were recorded, only the principal reason for admission was used to calculate mortality and morbidity rates. When the diagnosis, outcome or other data are missing in the registers, this was noted and analysis with regard to other variables was done and included in the total number of admissions, but was excluded from the calculation of mortality and morbidity rates. Chi-square test was applied to test for significance of difference between proportions and t-test was used to compare means of continuous variables.

The data were entered into a computer and analyzed using SPSS version 11 statistical software package. Since the study subjects are only patients admitted under emergency ward, the finding can not be generalized to all pediatric patients admitted to the hospital. Besides, the data that were missed from the registry posed additional limitation to this study to describe the quality of care with strong evidence.

\section{Results}

There were a total of 2,522 admissions to the emergency ward in one year and 1,459 (57.9\%) of these were males and $1,063(42.1 \%)$ were females. The male to female ratio was 1.4. Age was recorded in the register for 2,469 (98\%) admissions, of which 1,151(46.4\%) were below 1 year, 780 (31.6\%) were between 1-5 years of age and 553 (22.4\%) were above 5 years of age. Almost $76.5 \%$ of the admissions were less than or equal to 5 years of age. The mean age for male and female study subjects was 2.9 and 2.8 years respectively. The maximum age was 13 years for both sexes.

There were 2,471 admissions for which diagnosis was recorded in the register. Diagnosis was not recorded for 51 (2\%) of the admissions. Table 1 shows that severe pneumonia, which accounted for $38.6 \%$ of the total admissions, was the most common cause of admission to the pediatric emergency ward of TAH. Sixty percent of all admissions were due to severe pneumonia (38.6\%), meningitis (7.2\%), sepsis (5.6\%), heart diseases (4.6\%) and tuberculosis (4.3\%).

Table 1: Percentage of admissions to the Pediatric Emergency Ward of TAH, Addis Ababa, Ethiopia (September 2002-August 2003)

\begin{tabular}{|c|c|c|}
\hline Diagnosis & $\mathbf{n}$ & $\%$ \\
\hline Severe pneumonia & 937 & 38.6 \\
\hline Meningitis & 182 & 7.2 \\
\hline Sepsis & 141 & 5.6 \\
\hline Heart diseases* & 115 & 4.6 \\
\hline Tuberculosis & 109 & 4.3 \\
\hline Severe malnutrition** & 89 & 3.5 \\
\hline Gastroenteritis & 87 & 3.4 \\
\hline Head injury & 58 & 2.3 \\
\hline Relapsing fever & 54 & 2.1 \\
\hline Fractures & 47 & 1.9 \\
\hline Bronchial asthma & 43 & 1.7 \\
\hline Malignancies ${ }^{\star \star \star}$ & 43 & 1.7 \\
\hline Severe malaria & 36 & 1.4 \\
\hline Burns & 29 & 1.1 \\
\hline Septic arthritis & 25 & 1.0 \\
\hline DKA $A^{\star \star \star \star}$ & 24 & 1.0 \\
\hline Pediatric AIDS & 23 & 0.9 \\
\hline Anemia (all causes) & 23 & 0.9 \\
\hline Cellulites & 21 & 0.8 \\
\hline Soft tissue injury & 17 & 0.7 \\
\hline Osteomyelitis & 16 & 0.6 \\
\hline Seizure disorder & 15 & 0.6 \\
\hline Submandibular abscess & 14 & 0.6 \\
\hline Acute febrile illness & 13 & 0.5 \\
\hline Croup syndromes & 11 & 0.4 \\
\hline No diagnosis recorded & 51 & 2.0 \\
\hline Others & 263 & 10.4 \\
\hline Total & 2,522 & 100 \\
\hline
\end{tabular}

* Heart diseases included congenital \& acquired heart problems with or without CHF; ** Severe malnutrition included marasmus, kwashiorkor and marasmic kwashiorkor; ${ }^{* * *}$ Malignancies included leukemia, lymphomas, brain tumors, sarcomas, Wilm's tumor, and retinoblastoma; DKA = diabetic ketoactidosis 
Outcome of admission was recorded for 2,149 (85.2\%) of the patients. Out of these, 1,789 (70.9\%) were discharged improved, 373 (14.8\%) had unknown outcome or no record of the outcome and $360(14.3 \%)$ died in the Hospital. The majority (71\%) of patients with head injury were discharged improved, followed by severe pneumonia (78.1\%) and gastroenteritis (67.4\%), Table 2.
There were 360 deaths recorded during the study period. Table 4 revealed that severe pneumonia was responsible for $41.9 \%$ of the total deaths; and $75.2 \%$ of all deaths were due to severe pneumonia, meningitis, sepsis, severe malnutrition and gastroenteritis.

Table 2: Outcome of admissions by diagnosis in the casualty ward of TAH, Addis Ababa, Ethiopia, Sept. 2002Aug 2003

\begin{tabular}{lllll}
\hline \multirow{2}{*}{ Diagnosis } & \multicolumn{3}{c}{ O U T C O M E } & \multirow{2}{*}{ Totals No (\%) } \\
\cline { 2 - 4 } & Improved No (\%) & Died No (\%) & Unknown No (\%) & \\
\hline Severe pneumonia & $754(78.1)$ & $151(15.6)$ & $61(6.3)$ & $966(38.3)$ \\
Meningitis & $130(71.4)$ & $37(20.3)$ & $15(8.2)$ & $182(7.2)$ \\
Sepsis & $74(53.2)$ & $46(33.1)$ & $19(13.7)$ & $139(5.5)$ \\
Heart diseases & $90(76.9)$ & $14(12.0)$ & $13(11.1)$ & $117(4.6)$ \\
Tuberculosis & $93(85.3)$ & $14(12.8)$ & $2(1.8)$ & $109(4.3)$ \\
Severe malnutrition & $66(74.2)$ & $16(18.0)$ & $7(7.9)$ & $89(3.5)$ \\
Gastroenteritis & $62(67.4)$ & $21(22.8)$ & $9(9.8)$ & $92(3.6)$ \\
Head injury & $44(71.0)$ & $2(3.2)$ & $16(25.8)$ & $62(2.5)$ \\
All others & $476(62.1)$ & $59(7.7)$ & $231(30.2)$ & $766(30.4)$ \\
\hline Total & $\mathbf{1 7 8 9 ( 7 0 . 9 )}$ & $\mathbf{3 6 0 ( 1 4 . 3 )}$ & $\mathbf{3 7 3 ( 1 4 . 8 )}$ & $\mathbf{2 5 2 2 ( 1 0 0 )}$ \\
\hline
\end{tabular}

There was no significant difference in mortality rate between females (15.3\%) and males (14.1\%) $(95 \%$ CI of OR 0.89-1.41). However, as indicated in Table 3 there is a significantly higher death rate among infants less than 1 year of age (19.4\%) than children between 1-5 years of age $(11.5 \%)$ and older than 5 years of age $(8.5 \%)(\mathrm{P}$
$=0.001)$. The chi-square test for linear trend showed that there is significant decrease in the risk of death as we move to the older age categories (Chi-square for trend test $=41.26, \mathrm{P}<0.001)$.The overall mortality rate in the Pediatric Emergency Ward of TAH was 14.3\%.

Table 3: Age specific mortality rates among 2,484 admissions to emergency ward of TAH, Addis Ababa, Ethiopia, September 2002-August 2003

\begin{tabular}{llcclcccc}
\hline \multirow{2}{*}{$\begin{array}{l}\text { Age } \\
\text { group }\end{array}$} & Admissions & Deaths & Mortality & $\begin{array}{l}\text { Odds } \\
\text { ratio }\end{array}$ & $\begin{array}{l}\text { Lower } \\
\text { limit }\end{array}$ & $\begin{array}{l}\text { Upper } \\
\text { limit }\end{array}$ & X $^{2}$ trend & P-Value \\
\hline$<1 \mathrm{yr}$ & $1151(46.4)$ & 223 & 19.4 & 1 & & & $<0.0001$ \\
$1-5 \mathrm{yr}$ & $780(31.4)$ & 90 & 11.5 & 1.84 & 1.41 & 2.39 & 41.26 & \\
$>5 \mathrm{yr}$ & $553(22.2)$ & 47 & 8.5 & 2.58 & 1.60 & 3.60 & & \\
\hline Total & $\mathbf{2 4 8 4 ( 1 0 0 )}$ & $\mathbf{3 6 0}$ & $\mathbf{1 4 . 5}$ & & & & & \\
\hline
\end{tabular}

Note: For 38 cases age was not recorded and these have been excluded from this analysis

Table 4: Major causes of death among 360 patients who died in the causality ward of TAH, Addis Ababa, Ethiopia, September 2002-August 2003

\begin{tabular}{lcc}
\hline Diagnosis & Number & Percentage \\
\hline Severe pneumonia & 151 & 41.9 \\
Sepsis & 46 & 12.8 \\
Meningitis $^{1}$ & 37 & 10.3 \\
Gastroenteritis $^{\text {Severe Malnutrition }}$ & 21 & 5.8 \\
Tuberculosis $^{2}$ & 16 & 4.4 \\
Hear diseases $^{3}$ & 14 & 3.9 \\
Pediatrics AIDS & 14 & 3.9 \\
Hepatitis & 9 & 2.5 \\
Anemia (all causes) & 5 & 1.4 \\
All Other causes & 4 & 1.1 \\
\hline Total & 34 & 11.9 \\
${ }^{1}$ Meningitis included all causes except TB meningitis \\
${ }^{2}$ Tuberculosis included pulmonary and extra-pulmonary TB \\
${ }^{3}$ Heart diseases included congenital and acquired heart \\
problems with or without congestive hear failure.
\end{tabular}

The mean and maximum hospital stays for both sexes were 5 days and 33 days, respectively. The minimum hospital stay was one day for both sexes. The longest mean hospital stay was for meningitis (8.7 days) and the shortest for sepsis and severe pneumonia (4 days each), Table 5. There was significant difference in the duration of hospital stay between meningitis and severe pneumonia $(\mathrm{P}<0.001)$.

\section{Discussion}

The current analysis revealed that there were 2,522 admissions under the emergency room in one year which is almost twice of what was reported in 1970-71(7).This indicates that the number of admissions to the Pediatrics Department of TAH has greatly increased because of the population increase in the past 20 years (5). On the other 
Table 5: Comparison of duration of hospital stay between meningitis and severe pneumonia cases admitted to Casualty Ward of TAH, Addis Ababa, Ethiopia, September 2002-August 2003)

\begin{tabular}{|c|c|c|c|c|c|c|c|}
\hline \multirow[t]{2}{*}{ Diagnosis } & \multirow[t]{2}{*}{ Number } & \multirow{2}{*}{$\begin{array}{l}\text { Mean } \\
\text { hospital }\end{array}$} & \multirow{2}{*}{$\begin{array}{l}\text { Std } \\
\text { Deviation }\end{array}$} & \multirow{2}{*}{$\begin{array}{l}\text { T-test } \\
\text { value }\end{array}$} & \multicolumn{2}{|c|}{$95 \% \mathrm{Cl}$} & \multirow{2}{*}{$\begin{array}{l}\text { Mean } \\
\text { difference }\end{array}$} \\
\hline & & & & & Lower & Upper & \\
\hline Meningitis & 182 & 8.76 & & 13.7 & 4.01 & 5.36 & \\
\hline Severe Pneumonia & 966 & 4.07 & 5.42 & & & & \\
\hline Total & 1,148 & & 3.41 & & & & \\
\hline
\end{tabular}

hand, the number of hospital beds, rooms and other facilities have not been changed over the past 30 years, contributing to the congestion and discomfort of patients in causality ward.

The four leading causes of admission in this series are severe pneumonia (38.6\%) which was defined clinically based on WHO case definitions (8), meningitis (7.2\%), sepsis (5.6\%) and heart diseases (4.6\%). In the previous study, malnutrition (18.5\%), neonatal disorders (16.8\%), diseases of the respiratory system including pneumonia (10.6\%) and infective and parasitic diseases (9.8\%) were the four major causes of admission to all the wards (7). Severe pneumonia, which is the leading cause of admission in this analysis, was the third most common cause of admission in the previous study in the same hospital and the second most common cause in a district hospital in Eldoret, north-western Kenya in 1991-93 and was the cause of $36.4 \%$ hospital admissions to the Coast Province General Hospital in Mombasa, Kenya in 1960 $(7,9,10)$. Meningitis, which is the second most common cause of admission in our analysis, was the $6^{\text {th }}$ common cause of admission in the previous study in TAH, as well as the one at Eldoret. The Kenyan studies described here are based on district and province hospital data which may be a reason for observed difference from our study findings which is from specialized teaching hospital data source. However, infectious causes still remained important causes of hospital admission in children in different places and time. World wide also pneumonia is the most common cause of childhood deaths which accounts for $19 \%$ of all deaths. In our study also pneumonia is an important cause of admissions and deaths. Measles, which was the $5^{\text {th }}$ common cause of death in TAH in the previous analysis (7) and the $4^{\text {th }}$ most common cause of pediatric admissions to the Eldoret District Hospital in Kenya (9),, was not seen at all in our study. In fact the patients in the Kenyan hospitals $(7,9)$ could have different characteristics from a specialized teaching hospital like TAH. The absence of a single registered measles case in this study indicates satisfactory measles control through routine and supplemental immunization activity in the country that reduced deaths and illnesses due to measles. This finding is an evidence for effectiveness of use of vaccines as a strategy to reduce childhood illnesses if implemented appropriately. Further study is warranted to identify the commonest causes of pediatric meningitis, sepsis and pneumonia to determine if we could use other available vaccines in preventing childhood deaths and illnesses due to infectious causes.

The overall mortality rate in the pediatric emergency ward of TAH was $14.3 \%$. In Lester's analysis of adult medical admissions in Addis Ababa in 1971-75, the overall mortality rate was reported to be $14 \%$ (11). In the previous study of pediatric admissions to $\mathrm{TAH}$, which included all the wards, and the Diarrhea Training Unit, the overall mortality rate was $20.8 \%$ (7) which is somehow higher than our finding mainly because it included the neonatal ward. Although there is difference in the study populations, the overall observations indicate that the death rate in the hospitals has not changed markedly after several decades thus underlining the need to evaluate and monitor our interventions in terms of reducing childhood deaths.

Since hospital stay before death couldn't be found from the registration book or patient charts, it was not possible to assess the quality of care. However, the observed high overall mortality rate in the Pediatric Emergency Ward of TAH may be partly explained by the ever-increasing patient flow to TAH and points to the need of improving the capacity of the facility aiming at improving of the quality of patient care..

The four admission diagnoses with the highest case fatality rates in the current study are pediatric AIDS (39.1\%), sepsis (33.1\%). gastroenteritis (24.1\%) and meningitis (20.4\%). In the Eldoret study (9), the four diseases with the highest case fatality rates were prematurity (43.5\%), sepsis (27\%), hepatitis (20\%) and severe anemia (17\%). Pediatric AIDS, which was nonexistent in the other studies $(6,7,9,10)$, has emerged as the diagnosis with the highest case fatality rate in our study. On the contrary, measles which was one of the important causes of admission in the previous study (7) was not found in the list of causes of admission in this study indicating evidently some change of diseases trend.

The mean hospital stay for sepsis was observed to be unusually short as compared to meningitis. This may be because the diagnosis is made based on the clinical picture of patients and those cases diagnosed as sepsis my not have true sepsis. The hospital stay for meningitis is longer than pneumonia which may be due to the neurological complications associated with meningitis that needs longer period of treatment. (12). However, further study is needed to verify the finding. 
More lives can be saved by enabling mothers to recognize childhood diseases (13). Meanwhile improved training of health workers would help ensure rapid diagnosis of life threatening disease in young children in areas where the major cause of deaths are avoidable. (14).

\section{Conclusion and recommendation}

The unrelenting high overall mortality rate seen in the pediatric emergency room of TAH despite the development of new technologies in the last decades warrants the need of improving the quality in terms of facility and staffing.

As major causes of hospital admission and deaths are pneumonia, meningitis, sepsis and gastroenteritis which are preventable and treatable with available medical technology, there is a room to reduce childhood deaths and illnesses by improving case detection and management. Preventable infectious diseases are still persisting as important causes for childhood illness and deaths across several decades in the country $(6,7,15)$. Considering the impact of large scale intensified measles vaccination in the country, surveillance of etiologic agents of these diseases and identifying and introducing available effective technologies that could result in reducing childhood morbidity and mortality is of paramount importance. Intensifying prevention and case management of pediatric HIV/AIDS is also time-worthy in reducing childhood deaths (16).

The death rate decreases significantly as the age of children increases. Parents should be informed of the importance of early treatment seeking for any health problem seen in their young children. Health professionals should make timely case detection, appropriate management and follow up of young children.

The hospital record keeping system also desires great improvement to help obtain complete information on the patients registered for further similar studies.

\section{Acknowledgments}

We would like to express our gratitude to the casualty ward nurses and the hospital staffs in general for their valuable support.

\section{References}

1. Johan Giesecke. Modern infectious disease epidemiology, 1994;1-19.

2. Ivanhoe Newswire. Preventing Childhood Deaths. Lancet, 2003;361:2226-2234.

3. Shamebo D, Sandstrom A, Muhe L, Frej L, Kantz T, Lonnberg G,Wall S. The Butajira Project in Ethiopia: A nested case referent study of under five mortality and its public health determinants. Bull WHO 1993;71:389-96.

4. Ministry of Health Federal, Democratic Republic of Ethiopia. National Technical Guideline for Integrated Disease Surveillance and Response (IDSR), June 2003;1-4.

5. Federal Democratic Republic of Ethiopia. Health and Health Related Indicators, 1991;1-17.

6. Habte-Gabr E, Girma B, Mehrete M, Mehari A, Tekle E, Belachew K, Amera B. Analysis of admissions to Gonder Hospital in North Western Ethiopia, 1971. Ethiop Med J. 1976;14:49-59.

7. Tafesse, B. Analysis of admissions of the EthioSwidish Pediatric Clinic (1970-1971). Ethiop Med J. 1973;11:3-11.

8. Management of the child with a serious infection or severe malnutrition. WHO, GENEVA 2000.

9. Menge I, Esamai F, Van Reken D, Anabwani G. Pediatric morbidity and morality at the Eldoret District Hospital, Kenya. E Afr Med J 1995;72(3)165-169.

10. Turner, P.P. The pattern of disease as seen by medical admissions to Coast Province General Hospital in 1960. E Afr Med J. 1962;39:121-135.

11. Lester F.F, Tsega E. The pattern of Adult medical Admission in Addis Ababa, Ethiopia. E Afr Med J. 1976;53(11):620-34.

12. Cabellos C, Viladrich PF, Verdaguer $\mathrm{R}$, et al, Clinical Infectious Disease 1995;20:1164-168.

13. Richard J. Sagall, Pediatrics for Parents, September 2004.

14. http://www.who.int/inf-new/child.htm. Childhood Diseases, 25 October 2006.

15. Fantahum M. Patterns of childhood mortality in three districts of North Gonder Administrative Zone. A community based study using the verbal autopsy method. Ethiop Med J. 1998;36:71-81.

16. Guidelines for the clinical management of pediatric HIV infection in Ethiopia. First Edition, 2000. 